\title{
Intraoknlares Sarkom.
}

\author{
Vom
}

Kaiserlichen Rate Dr. Theodor Ballaban, Augenarzt in Lemberg.

Mit Taf. V, Fig. 1-5.

Durch zahlreiche einzelne Mitteilungen und einzelne zusammenfassende Bearbeitungen ist das intraokulare Sarkom eingehend in seiner Pathologie erforscht worden ${ }^{1}$ ). Im Verlauf des Sarkoms wurden auch auf Grund der vorliegenden Erfahrungen bestimmte Abschnitte unterschieden, welche der Regel nach in bestimmter Reihenfolge, häufig in bestimmter Zeitfolge ineinander iubergehen. Wir kommen später (S. 75) noch ausführlicher darauf zuriick, und ich führe daher nur die Einteilung nach Fuchs an: I. Stadium des reizlosen Wachstumes der Geschwulst, II. Stadium des sekundären Glaukoms oder selten von Iridocyclitis, III. Stadium der Perforation.

Neben diesem nunmehr als typisch geltenden Verlaufe des intraokularen Sarkoms findet man in der Literatur eine ganz kleine Anzahl von Fällen, welche bezüglich Auftreten und Verlauf eine ganz andere Erscheinungsart darbieten. Da ich einen dieser abweichenden Kategorie angehörigen Fall zu beobachten Gelegenheit hatte, so möchte ich an dieser Stelle ausführlicher meine darauf bezüglichen Untersuchungen mitteilen.

\section{Fall.}

Im Herbste 1900 wurde ich in die weibliche Strafanstalt zu Lemberg behufs Untersuchung einer 29jährigen Frau gerufen.

Dieselbe klagte darüber, dass das Sehvermögen des linken Anges abnehme, nachdem sie kurze Zeit vorher anch bemerkt hatte, dass auf der äussern Seite des linken Augapfels eine kleine Geschwulst sich befinde,

1) E. Fuchs, Das Sarkom des Uvealtractus. Wien 1882.

R. Kerschbaumer, Das Sarkom des Auges. Wiesbaden 1900. 
welche allmäblich an Grösse zunahm, ohne ihr irgendwelche Schmerzen zu verursachen. Die von mir damals vorgenommene Untersuchung ergab tatsächlich eine bohnengrosse bläulich-schwarze Gesehwulst, welche der Sklera lateral aufsass und einige Millimeter rom Hornhautrande entfernt begann. Dieselbe war mit der Unterlage fest verwachsen, ihre Oberflüche war glatt, dabei war sie von der Bindehaut, welche über ihr überall verschiebbar war, vollkommen bedeckt. Dem äussern Aussehen nach musste die Geschwulst als ein Melanosarcoma angesprochen werden.

Die Augenspiegeluntersuchung ergab entsprechend dem Sitze der Neubildung eine scharf umschriebene Netzhautablösung, welche mit ihrem hintern Rande die Macula nicht erreichte. Spannung des Auges war normal. Die zentrale Sehschärfe betrug $5 / 15$. Über das Wie und Wann der ersten Entstehung der Geschwulst wusste die wenig intelligente Patientin nichts Näheres anzugeben.

Den Vorschlag der Enucleation wies sie entschieden zurück.

Im Februar des nächsten Jahres (1901) bot sieh mir Gelegenheit dar, sie zum zweitenmal zu untersuchen.

Ich bemerkte, dass im Verlaufe der verflossenen fünf Monate die Geschwulst rasch an Ausdehnung zugenommen hatte.

Da die Sehschärfe dieses Auges bedentend vermindert war und sich bloss auf Erkennen der Finger knapp vor dem Auge beschränkte, entsehloss sich die Patientin endlich, sich der Enucleation zu unterziehen, welche ich auch sofort vornahm.

Der kurz vor der Enucleation aufgenommene Befund war folgender.

Die Lidspalte des linken Auges ist sehr stark verengt, indem das obere Lid stark herabgedrängt ist. Am engsten erseheint dieselbe in ihrer äussern Hälfte, wo ihre Breite auf kaum $1 \mathrm{~mm}$ reduziert ist. Je mehr gegen die innere Hälfte, desto weiter wird die Lidspalte; "im allgemeinen erreicht sie aber kaum die Hälfte der Weite der normalen rechten Lidspalte. Die Haut des obern Lides ist prall gespannt, bläulich durchscheinend, unter ihr sind zahlreiche Venen deutlich sichtbar. Der in allen seinen Bewegungen stark beschränkte Augapfel ist bei gerader Blickrichtung des rechten Auges derartig medialwärts gewendet, dass die Ablenkung an der Cornea gemessen ungefähr 5 bis $6 \mathrm{~mm}$ beträgt. Hebt man das obere Lid mit den Fingern vom Bulbus ab, so sieht man, dass die ganze laterale Hälfte der Lidspalte von einem rötlichen, stellenweise braunen oder bläulich weissen Tumor, dessen recht glatte Oberfläche von zahlreichen Gefässen bedeckt ist, erfüllt ist. Bei möglichst starker Erweiterung der Lidspalte sieht man, dass neben dieser Neubildang, welche die Grösse einer Walnuss besitzt, einige kleinere knollenförmige Erhebungen sich befinden, die unter der untern und obern Übergangsfalte, und zwar in der Gegend des änssern Lidwinkels sich vordrängen. Bei Betastung erscheint der Tumor ziemlich elastisch. Soviel man eruieren kann, scheint dieser Tumor in keiner Verbindung mit der knöchernen Orbitalwand zu stehen. Am Augapfel fest aufsitzend, ist er auf demselben unverschiebbar, dagegen ist die Bindehaut, welehe nahe dem Hornhautrande sulzig verdickt ist, darüber frei verschiebbar. Die Pupille reagiert auf Licht etwas träger. Cornea normal durehsichtig, ebenso die vordere Kammer normal tief. Regenbogenhaut unverändert. 
Ophtlualmoskopisch lässt sich aussen unten eine kuglige, weisslich graue Geschwulst unter der Netzhaut erkennen, welche lateral in der peripheren Grenze des ophthalmoskopischen Gesichtsfeldes, in einer Breitendimension von fast einem Netzhautquadranten, beginnend, gegen die Pupille zu sich langsam verschmälert. Dadureh gewinnt die Geschwulst, von vorne gesehen, die Gestalt eines abgestumpften Kegels, dessen Basis in der Gegend des Ciliarkörpers sich befindet, dessen abgestumpfte Spitze eben die Papille erreicht. Die Ränder dieses Kegels gehen steil in die weit nach oben und unten flach abgehobene Netzhaut über. Die Netzhantgefässe sind im Bereiche der abgehobenen Netzhaut sehr dünn, besonders die Arterien. Die iibrige in überwiegend grosser Ausdehnung noeh anliegende Netzhaut erscheint im ganzen etwas getrübt, die auf ihr vorhandenen Venen stark geschlängelt und stellenweise in ihrem Verlaufe wie unterbrochen. Die Sehnervenpapille ist trüb rot, erscheint geschwollen, doch lässt sich das nicht deutlich erkennen, da die angrenzende Netzhaut lateral noch flach abgelöst erscheint. Alle Venen sind sehr weit, dagegen die Arterien enger.

Bei Augenbewegungen ist es auffallend, dass die abgelöste Netzhaut keinerlei Flottieren zeigt.

Bei der Exstirpation des Tumors mit dem Bulbus zeigt es sich, dass der Tumor zum Teil auch die Augenmuskeln einbezogen hat, aber überall wie mit einer Kapsel gegen die Orbitalgebilde sich abgrenzt, so dass er sich ganz glatt ausschälen lässt. Dabei erfolgt eine starke Blutung aus den zahlreichen weiten Gefässen.

Härtung in $10 \%$ Formalinlösung; wobei der Augapfel etwas collabiert.

\section{Makroskopischer Befund.}

Das Neoplasma liegt der lateralen Augapfelhälfte an, mit seinem vordern Rande ungefähr $8 \mathrm{~mm}$ vom Hornhautrande abstehend, mit seinem hintern Rande den Sehnerven berührend. Die Geschwulst ist derb, trägt mehrfache halbkugelige, sekundäre Erhebungen, so dass sie ein grobhöekeriges Aussehen gewinnt, ist aber überall vollkommen glatt.

Nach Vollendung der Härtung in Alkohol wird der Augapfel oben und unten abgekappt, dann in toto samt Neoplasma in Celloidin eingebettet und in seinen mittlern Teilen mit dem Mikrotom in eine lückenlose Serie, ungefähr horizontaler Totalschnitte zerlegt.

Die Sehnervenpapille wird schliesslich noch separat in gleicher Richtung geschnitten. Die Durchschnittsstellen der Geschwulst zeigen ein grob marmoriertes Aussehen, indem in die im allgemeinen dunkle Geschwulst hellgelbliche Gewebsinseln eingetragen sind. An mittlern Durchschnitten (s. Fig. 1) sieht man, dass nur in einer Längenausdehnung von kaum $1 \mathrm{~cm}$ und nur ungetähr $4 \mathrm{~mm}$ Dickendurchmesser eine Neubildung von heller Farbe und dichtem Gefüge in der Chorioidea besteht, ïber der die Netzhaut leicht gefaltet, aber nicht beträchtlich abgehoben erscheint. Die Sklera ist in der Mitte der Aderhautgeschwulst in kleiner Ausdehnung durchbrochen, so dass die letztere in ununterbrochener Kontinuität mit der epibulbären Geschwulst steht. Die übrige Netzhaut völlig normal anliegend, Glaskörper 
normal, vordere Kammer mässig tief. Die Sklera erscheint im Bereiche der vordern Hälfte der epibulbären Geschwulst eingedrückt, ist aber, wie gesagt, mit Ausnahme einer kleinen Stelle, als deutliche Grenze zwischen extraokularer und intraokularer Geschwulst sichtbar.

\section{Histologischer Befund.}

Die Aderhautgeschwalst besteht aus in alien Richtungen sich kreazenden Zügen spindelförmiger Zellen, welche oft durch Nester und Züge rundlicher Zellen durchbrochen sind, so dass am Schnitte ein leicht marmoriertes Aussehen besteht. Sie ist von sehr spärlichen dünnwandigen Gefässen durchzogen, in deren Nachbarschaft an manchen Stelien Haufen intensiv gefärbter, rundlicher Kerne angesammelt sind. Nur an wenigen Stellen ist zwischen den Zellen eine zartfaserige bindegewebige Zwischensubstanz eingelagert. In der grössten Ausdehnung ist die Neubildung pigmentlos, nur an oberflächlichen Partien sind einige grössere Herde pigmentierter Zellen eingetragen, sowie in der Nachbarschaft weniger Gefässe. Nach vorn zu grenzt sich die Neubildung ungefähr $10 \mathrm{~mm}$ hinter der Ora serrata recht scharf $a b$, indem sie sich wie ein Keil (am Schnitte) zwischen die verdickte Lamina fusca chorioideae und die innern Chorioidealschichten eindrängt. Die erstere ist in grosser Ausdehnung sehr mächtig entwickelt und pigmentiert.

Die Aderhaut selbst ist nach vorn von der Gesehwulst ganz dünn, derb bindegewebig, atrophisch und von nur spärlichsten Gefässen durchzogen. Nach hinten (gegen den Sehnerven) ist die Hauptmasse der Geschwulst undeutlich abgegrenzt, die hier von weiten, strotzend gefüllten Blutgefässen (vorwiegend Venen) durchzogene Chorioidea stark gefaltet und fast rechtwinklig abgehoben (siehe Fig. 2). Die zwischen der Geschwulst und dem Sehnerven sich befindende Chorioidea ist auch etwas bindegewebig atrophiert, aber recht zahlreiche dickwandige Gefässe enthaltend. Die innern Schichten der Chorioidea sind bis znm Sehnerven gefaltet, die Lamina fusca fehlt bis dahin, und schiebt sich von der Aderhautgeschwulst an ihrer Stelle eine flache zellige Gewebslage gleicher Beschaffenheit, wie der Haupttumor, bis fast unmittelbar an den Sehnerven heran, vor. Die Sklera ist hier gleichfalls gefaltet, die einspringenden Winkel sind von der Neubildung erfüllt, so dass die Chorioidea die skleralen Falten nicht mitmacht.

Das Pigmentepithel ist im ganzen Umkreis der Aderhautgeschwulst unregelmässig, aus verbildeten, mitunter mehrschichtig angeordneten Zellen bestehend, und schiebt sich mit der Lamina elastiea chorioideae allseitig noch eine grosse Strecke auf die Geschwulst hinauf. Fast überall ist aber die Geschwulst deutlich durch die Lamina elastica oder durch eine dünne Lage faserigen Bindegewebes davon geschieden. Nur an einer Stelle in der Mitte der Geschwulst ist das Pigmentepithel gewuchert und grenzt als ein- bis zweischichtige, fast völlig pigmentlose, hyalinähnliche geschichtete, von spärlichen Zellen durchzogene Masse, die der Geschwulst anliegt, dieselbe ron der Netzhaut ab, bzw. verbindet beide, eine Neubildung, die der Kapselkatarakt nicht unähnlich ist.

Die Netzhaut ist zwischen Sehnerv und Geschwulst nur flach dureh dichte Eiweissmasse abgehoben. Auf den ersten Blick (Fig. 1) scheint es, 
dass sie über der Geschwulst sackförmig von der Unterlage abgelöst sei. Bei genanerer Betrachtung ergibt sich aber das Irrtümliche der Anschauung, und man erkennt, dass es sich um eine eigentümliche Form von eystoider Hohlraumbildung in der Netzhauthandelt. Wenn man auf einem der Schnitte die Netzhaut von vorn nach hinten verfolgt, so ergibt sich folgender Befund.

Ora serrata normal. Bald dahinter ist die Netzhaut dünner, die Stäbchenzapfen fehlen, bzw. sind durch tropfenähnliche ungefärbte Gebilde angedeutet, die beiden Körnersehichten sind zu einer verschmolzen und von spindelförmigen Zellen durchzogen. Ganglienzellen fehlen. Alle innern Sehichten sind bindegewebig verdickt und entartet, tragen zapfen- und kammartige Erhebungen bindegewebiger Struktur. Nervenfasern nicht nachweisbar. Am Beginne der Geschwulst ist die Netzhaut durch mächtige Bindegewebswucherung in den innern Schichten verdickt (siehe Fig. 3), bei weiterm Verlaufe anch die verschmolzenen Körnerschichten mächtig verbreitet. Die Körner sind gxösstenteils verschwunden, an ihrer Stelle befinden sich reichliche Ziüge ovaler oder spindelförmiger Zellen. In diesem letzten Gewebe treten kleine rundliche tropfenähnliche Hohlräume erst einzeln, dann gehäuft auf (Fetttropfen?), die allmählich zu grössern Hohlräumen zusammenschmelzen (siehe Fig. 4), so dass ihre Masse die des zelligen Gewebes mehrfach übertreffen; endlich treten grosse rundliche Hohlräume auf, deren Zentrum von geronnenen Eiweissmassen erfüllt ist, welehe gegen die Wand zu von reichlichen ungefärbten tropfenähnlichen Gebilden verschiedenster Form abgegrenzt ist; plötzlich teilt sich die Netzhaut in eine dieke äussere Lage, welche aus grösstenteils ausgezogenen Zellen mit bindegewebiger Zwischenmasse besteht, in der aber noch sekundäre Hohlräume der früher beschriebenen Beschaffenheit sich finden, und in eine innere, drei- bis fünfmal so dicke Schicht, an der überall die innere Körnerschicht deutlich nachweisbar ist. Gegen den hintern Tumorrand ist die Grenze des einer grossen Cyste ähnlichen HohIraumes scharf, und bildet das äussere (chorioideale) Netzhautblatt eine kleine Duplikatur (Fig. 2). Das äussere Blatt ist mit der Geschwulstoberfläche in kleiner Ausdehnung innig verwachsen, nur spärliche Pigmentepithelzellen trennen beide, aber die Geschwulst selbst grenzt sich scharf gegen dasselbe ab (Fig. 4).

Der grosse cystoide Hohlraum ist mit geronnenen Eiweissmassen gefüllt, in die spärliche zum Teil pigmentierte Zellen, sowie unregelmässige tropfenartige Lücken eingelagert sind.

In der ganzen Umgebung der Gesehwulst sind die Stäbchen und Zapfen aufgequollen und zum Teil in Tropfen zerfallen.

In der medialen Hälfte ist die Netzhaut normal anliegend und ebenso wie die Chorioidea von normaler Struktur.

Nur nahe der Ora serrata findet sich eine unregelmässige Pigmenteinlagerung in der Umgebung einzelner Gefässe, ähnlich dem Befunde bei Retinitis pigmentosa, und erscheint daselbst auch das Pigmentepithel in Proliferation.

Der Sehnerv zeigt eine intensive Schwellung und Entzündung, vollkommen ähnlich einer Staungspapille bei Hirntumor. Die Papille ragt 
leicht pilzförmig hervor und besitzt eine trichterförmige zentrale Einsenkung. Ihr Stütz- und Bindegewebe ist vermebrt, kernreicher, die Nervenfasern nicht rarefiziert, das endotheliale Gewebe an der Innenfläche mächtig gewuchert, zarte Sprossen in den Glaskörper sendend.

Retrobulbär zeigt der Sehnerv eine mässige Kernvermehrung.

Der vordere Bulbusabsehnitt ist normal. Die Iris zeigt keine glaukomatöse Synechie, Ciliarkörper normal. Die Conjunctiva ist am Hornhautrande, besonders im Bereiche der Geschwulst mächtig verdickt. Sie besteht aus sehr lockerem ödematösen kernreichen Gewebe und reichlichen weiten Gefässen. Ihr Epithelbelag ist verdiekt und sendet stellenweise zapfenartige Fortsätze in die Tiefe.

Die epibulbäre Geschwulst unterscheidet sich nur dureh auffallend starke Pigmentierung einzelner Stellen von der intrabulbären. An einzelnen Stellen sind die Gesehwulstzellen in beginnender Nekrose. Gefässe spärlich. Die Oberfläche der Geschwulst ist durch eine derbe bindegewebige Kapsel abgegrenzt. Ein Augenmuskel (rectus lateralis) ist ganz in die Geschwulst einbezogen, seine Bündel atrophisch und durch Geschwulstelemente zersplittert. Die Sklera ist zwischen dem epi- und intrabulbären Tumor grösstenteils normal erhaiten. Nur in einem kleinen Bereiche ist die Sklera dicker, aufgefasert, von Gesehwulstnestern und Zügen durchsetzt. Durch solehe Züge steht in kleinen Bezirken die intra- und epibulbäre Neubildung in Kontinuität. Hier passiert eine grosse Vene, welche wohl als Vortexvene anzusehen ist, die Sklera in schräger Riehtung.

Wie aus der vorstehenden Beschreibung sich ergibt, liegt hier ein zum grössten Teil rein spindelzelliges, sehr pigmentarmes Sarkom der Chorioidea vor. Was an demselben besonders hervorzuheben ist: Es finden sich in der intraokularen Geschwulst keinerlei ausgesprochene Nekrosen, keine Stellen, welche auf beginnende Ernährungsstörungen in den zelligen Elementen hinweisen. Mit dieser intraokularen, relativ kleinen Geschwulst, welche weder zu Sekundärglaukom noch zu sekundärer Netzhautablösung geführt hatte, steht eine an Volumen und Ausbreitung die intraokulare Neubildung um ein vielfaches übertreffende extraokulare Neubildung in unmittelbarer Verbindung, welche von einer derben Bindegewebskapsel eingeschlossen ist. Letztere Geschwulst ist weitaus reichlicher pigmentiert und zeigt zahlreiche Herde beginnender Degeneration. Beide Tumoren stehen nur durch eine enge Brücke, welche anscheinend längs einer Vortexvene die Sklera durchsetzt, miteinander in Kontinuität. In histologischer Beziehung besteht zwischen beiden Neubildungen, wie bereits 
erwähnt, ausser dem grössern Pigmentreichtum und der beginnenden Nekrotisierung der extraokularen Neubildung keine Differenz.

Das Eigenartige dieses Falles besteht in dem Grössenverhältnis zwischen intra- und extraokularer Neubildung. Wie sich aus der Anamnese ergibt, hatte zur Zeit, als der Beginn der Sehstörungen die Kranke auf ibr intraokulares Leiden aufmerksam machte, die extraokulare Neubildung bereits eine sehr beträchtliche Grösse erreicht, hatte damals schon die intraokulare an Volumen bedeutend übertroffen. In der weitern Beobachtung trat in diesem relativen Grössenunterschiede keine wesentliche Veränderung, eher eine solche zu Gunsten der Grössenzunahme der extraokularen Neubildung ein. Vergleichen wir mit diesem Verlaufe den typischen Verlauf, so fehlt in unserem Falle das erste und zweite Stadium der Geschwulstbildung. Fuchs'), dem wir die erste eingehende und zusammenfassende Darstellung über das intraokulare Sarkom verdanken, stellt bekanntlich folgende vier Stadien des Verlaufes des intraokularen Sarkoms auf"): „Im ersten Stadium ist die Geschwulst noch klein und verrät sich nur bei der ophthalmoskopischen Untersuchung durch Abhebung der Netzhaut an der Stelle der Geschwulst." Bei weiterem Wachstum der Geschwulst stellt sich plötzlich Spannungszunahme, das Stadium glaukomatosum der Neubildung ein - zweites Stadium. Das dritte Stadium ist dasjenige des Hervorwucherns der Geschwulst nach aussen. Als einzige Varietät dieses typischen Verlaufes stellt Fuchs jene fest, dass das zweite entzündliche Stadium der Geschwulstbildung nicht die Symptome des Glaukoms, sondern die einer heftigen plastischen, Iridocyclitis zeigt.

Von diesem typischen Verlaufe, wie gesagt, unterscheidet sich mein Fall ganz wesentlich. Bei genauer Durchsicht der Literatur konnte ich nur 6 analoge Fälle finden, welche ich hier kurz zu referieren unternehme.

1. E. v. Forster ${ }^{3}$ ). Krankenvorstellung. Sarkom des Ciliarkörpers. Die Sklera zeigt sich durchwuchert. Knotenbildung auf derselben. Keine Netzhautabhebung. Dieser Buibus wurde enucleiert und von Königsberger anatomisch untersucht. Die Geschwulst war pigmentiert und ging vom obern innern Teil des Corpus ciliare ans, indem sie die Sklera durchbrach. Teilweise Dialyse der Iris. Keine Netzhautabhebung, obwohl die Chorioidea bis zum hintern Bulbusdrittel mit ergriffen war.

1) Fuchs, Das Sarkom des Uvealtractus. Wien 1882.

2) Fuchs, Lehrbuch der Augenheills. 1903. S. 417.

3) v. Forster, Sarkom des Ciliarkörpers. Münch. med. Wochenschr. 1889. S. 624 u. 712 . 
2. G. Freudenthal' $)$. Fall 11. Ludwig Grube, 62 Jahre, aus Göttingen. Rechtes Auge Sehstörungen seit vier Wochen. Chorioidealsarkom in der Gegend des Ciliarkörpers. Ophthalmoskopisch war die Papille auffallend rot. Netzhaut wie es scheint überall anliegend. Der Bulbus wurde enucleiert. An der Aussenseite des Bulbus ein circumscripter flacher, erbsengrosser Knoten, auch an der innern Seite eine episklerale Geschwulst.

3. Panas ${ }^{2}$ ). 50jährige Frau. Rechtes Auge. Vor zehn Jahren durch einen Fall auf die Schläfe erblindet. Am hintern Pol eine plattenförmige Erhebung, die die Papille frei lässt. Im Laufe der nächsten drei Jahre Glaukomanfälle und Exophthalmus. Bei der Enucleation traf man auf eine dem hintern Bulbusumfang aufsitzende, dem Sehnerven anliegende Geschwulst. Intraokular fand sich nur ein dünnes, plattenförmiges, Retina und Sklera anliegendes, gefässarmes, aber ziemlich pigmentreiches Spindelzellensarhom des hintern Poles der Chorioidea. Die übrigen Teile des Auges, mit Ausnahme der innern Schichten der benachbarten Retina, intakt.

4. Heine ${ }^{3}$ ) beschreibt unter dem Titel: „Ein seltenes Bild des Sarkoms" ganz kurz einen Bulbus, in dem das Sarkom, ohne zu Ablatio der Retina zu führen, sich zunächst sehr weit in der Aderhaut verbreitete, dann ist die Geschwulst nach aussen perforiert. Dieses Bild ist meinem ersten Falle sehr ähnlich.

5. Van Duyse ${ }^{4}$ ). "Es bestand ein wegen Altersstars nicht erkennbares Sarkom der Maculagegend, das auf dem Wege des Optikus und der hintern Ciliararterien in die Orbita hineingetreten war und sich hier erst drei Jahre später durch einen schnell zunehmenden Exophthalmus bemerkbar gemacht hatte. Die Geschwulst war ein Alveolarsarkom, von dem ein sehr kleiner, im Augeninmern gelegener Teil pigmentiert, der Rest sowie der orbitale Teil pigmentfiei war.

6. A. Groenouw ${ }^{5}$. Franz H., 28 Jahre alt, hat auf dem linken A u ge bis vor elf Wochen gut gesehen, dann Stoss gegen das Auge. Geringe Blutung and allmähliche Verschlechterung tes Sehens. Später am untern und obern Hornhautrand Geschwulstknoten. Schmerzhaft war das Auge nie. Anatomischer Befund: Ein eigentlicher intraokularer Tumor fehlt. Der Ciliarkörper erscheint vergrössert, sein Gewebe an den befallenen Stellen durch Geschwulstzellen ersetzt. Die Netzhaut liegt ïberall an. Papille excaviert. Der eine epibulbäre Knoten sitzt genau ausserhalb von der Ciliarkörpergesehwulst auf der Sklera, der andere am obern innern

1) A. Freudenthal, Über das Sarkom des Uvealtractus. v. Graefe's Arch. f. Ophthalm. Bd. XXXVII. 1. S. 137-184.

2) Panas, Sarcom chorioidien de la région de la macula avec propagation orbitaire. Arch. d'Ophtalm. Tom. XVI. No. 8. p. 465.

3) Heine, Neissers stereoskopischer medizinischer Atlas. Lief. 44.

4) Van Duyse, Sarcom chorioidien de la région de la macula avec propagation orbitaire. Arch. d'Ophtalm. Tom. XVI. No. 11.

๘) A. Groenouw, Ein Fall von alveolärem unpigmentiertem Flächensarkom des Ciliarkörpers. v. Graefe's Arch. f. Ophthalm. Bd. XLVII. S. 282. 
Hornhautrande. Ein deutlicher Zusammenhang zwischen dem intraokularen und den beiden epibulbären Tumoren lässt sich nicht mit voller Sicherheit nachweisen. Es finden sich in verschiedenen Schnitten Stiucke eines bindegewebigen Stranges, welcher die Sklera in schräger Richtung durchsetzend, sich vom intraokularen zum epibulbären Tumor erstreckte und der Wand eines vordern Ciliargefässes angehörte. Der. Zusammenhang mit jeder der beiden Geschwälste war an verschiedenen Präparaten zu erkennen. Doch waren in demselben die unpigmentierten endothelialen Geschwulstzellen nicht mit Sicherheit nachzuweisen.

Verfasser nimmt an, dass die Geschwulstzellen höchstwahrscheinlich längs eines vordern Ciliargefässes nach aussen gewuchert sind und so den epibulbären Knoten gebildet haben.

Im Anschlusse an vorstehende Fälle erlaube ich mir noch einen zweiten Fall, welcher in vieler Hinsicht analog ist, mitzuteilen. Diesen Fall beobachtete ich noch im Jahre 1891 und habe ihn später histologisch bearbeitet.

\section{Fall.}

S. J., 59 Jahre alt, liess sich wegen einer blauschwarzen Geschwulst am linken Auge in die Klinik aufnehmen. Dieses Auge war seit vielen Jahren nach einer Verletzung phthisisch, ohne jedoch die geringsten Beschwerden zu verursachen. Da der Kranke wenig intelligent war, hat er den Beginn der Erkrankung nicht beobachtet und ihr Wachsen erst seit einigen Wochen bemerkt.

Status praesens. Das Oberlid des linken Auges, dessen Lidspalte wenig geöffnet wird, ist leicht vorgedrängt, dagegen das untere eingesunken. Bei Hebung des obern Lides schiebt sich an der obern Hälfte des phthisischen Bulbus eine blanschwarze, teigig weiche Geschwulst vor, von der Conjunctiva glatt bedeckt, an dem Bulbus festsitzend. Die Gesehwulst reicht weit nach hinten, zu beiden Seiten etwa bis zum horizontalen Meridian des Bulbus und überlagert kappenförmig die phthisische Cornea. Die Geschwulst wurde exstirpiert, samt dem phthisischen Bulbus in Miillerscher Flïssigkeit gehärtet und mikroskopisch untersucht:

\section{Anatomiseher Befund an dem vertikalen Schnitte (siehe Fig. 5).}

Der Bulbus ist von oben nach unten stark flachgedrückt. Sagittale Achse $16 \mathrm{~mm}$, vertikaler äquatorialer Durchmesser $12 \mathrm{~mm}$. Die Sklera ist hochgradig gefaltet, in den vordern Partien von ziemlich normaler Dicke, in den hintern wesentlich verdickt. Die Linse, verkalkt und gesehrumpft, ist aus dem Schnitte herausgefallen. Die Geschwulst liegt der gleichfalls gefalteten obern Bulbuswand innig an und übertrifft in allen Dimensionen den Bulbus. Während im übrigen sich der Befund im Bulbus nicht von dem einer ältern Phthisis ohne wesentliche Entzündungserseheinungen unterscheidet, sieht man an der obern Bulbuswand einen lappigen, anscheinend aus mehreren kleinern Knoten bestehenden, dunkel pigmentierten Tumor, 
der durch eine zarte Geschwulstbrücke mit dem epibalbären Tumor im Zusammenhange steht, aber eine wesentlich abweichende histologische Struktur zeigt. Der epibulbäre Tumor ist ein grossenteils rein spindelzelliges Sarkom mit grössern Zügen und Inseln pigmentierter Zellen, das nur in den vordersten Partien ödematös erweicht, von Hämorrhagien (die erst intra operationem eingetreten $\mathrm{zu}$ sein scheinen) durchsetzt, in dem grössten Teile dagegen ohne Degenerationserscheinungen ist.

Die intraokularen Geschwulstknoten dagegen sind grösstenteils rein melanotiseh, aus dichtest pigmentierten, meist rundlichen Zellen bestehend, grösstenteils hochgradig nekrotisch. Kernfärbung ist nur an wenigen Stellen zu erzielen, grosse Teile sind in eine krümelige pigmentierte Masse zerfallen, die reichlichste, in Hämatoxylin dunkelblau gefärbte Körnchen (Kalk) und spiessförmige oder nadelartige schmale Spalten enthält, augenscheinlich Cholestearintafeln und Nadeln entsprechend. An einer Stelle finden sich in der Nachbarschaft solcher Inseln Riesenzellen. Die Knoten sind in das die Chorioidea und Netzhaut grösstenteils ersetzende straffe Bindegewebe eingetragen und senden an einzelnen Stellen zarte Ausläufer pigmentierter Zellen in dasselbe.

Während sich die vordern und hintern Partien der epibulbären Geschwulst ganz deutlich, aber ohne Bindegewebskapsel gegen die Umgebung abgrenzen, fehlt in den mittlern Partien diese Abgrenzung gegen die Sklera vollständig und ziehen sich hier Inseln und Stränge von Sarkomzellen in die Sklera hinein, um mit dem intraokularen Knoten in Verbindung zu treten. In den untern vordern Geschwulstpartien ist die Neubildung deutlich gegen die bedeckende Conjunctiva abgegrenzt, letztere stark aufgelockert und dicker, das Gewebe von reichlichen Gefässen und Zellen durchsetzt, nur an einer Stelle, gegen die obere Übergangsfalte zu, scheint die Neubildung im Begriffe die Conjunctiva zu perforieren; pigmentierte und unpigmentierte Geschwulstnester treten dicht an das Epithel heran, welches unlegelmässig, wie abgeschilfert, ist und aus verkümmerten Zellen besteht. Die epibulbäre Geschwulst enthält recht reichliche Gefässe, besonders weite Venen; die intraokularen Geschwulstknoten sind, wie das umgebende Bindegewebe, äusserst gefässarm.

Die Sehnervenpapille ist tief glaukomatös excaviert, aus ihrem Grunde erhebt sich die verbildete, abgelöste Netzhaut, die nach vorn zu sich ansbreitend in das straffe, die Linse nach hinten umgebende und mit den hochgradig verzerrten Ciliarkörperresten verbindende straffe Bindegewebe übergeht. Der Selunerv ist atrophiseh, dïnner, sein Glia- und Bindegewebe kernreich gewuchert, ebenso das Gewebe der Sehnervenseheiden.

Es handelt sich also in diesem Falle um kleine intraokulare Sarkomknoten in einem lange Zeit phthisischen Bulbus, welche in direkter Verbindung mit einem epibulbären, vielfach grösseren Sarkomknoten stehen. Hier lässt sich aus der Beschaffenheit der Knoten leicht die primäre Geschwulst erkennen. Die intraokularen Knoten sind fast völlig der Nekrose anheimgefallen, während die extraokularen nur in dem vordersten Anteile hydropisch verändert sind, nirgends 
nekrotische Herde aufweisen. Die intraokularen Knoten sind daher wohl als die primären, die epibulbären als sekundäre, in continuo aus den erstern entstanden, zu erklären. Weshalb hier die kleine Bulbushöhle nicht ausgefüllt ist rom Sarkomgewebe, also relativ früh die Perforation und epibulbäre Wucherung des Sarkoms erfolgt ist, ergibt sich wohl aus dem histologischen Befunde ohne weiteres. Der Boden, auf dem die primäre intraokulare Neubildung entstand, ist für die Ausbreitung einer Neubildung höchst ungünstig zu nennen. Das straffe, sklerosierte, ungemein gefässarme Bindegewebe, das die Geschwulst umgibt und Ursache der Phthisis ist, setzt der Ausbreitung der Geschwulst grossen. Widerstand entgegen, und bedingt auch, mangels ungenügender Blutversorgung und Ernährung, die Nekrose der Geschwulst selbst. Auf die Frage der Entstehung des Sarkoms im phthisischen Auge, oder Phthisis zufolge Sarkoms gehe ich hier nicht ein, da in letzter Zeit dieser Gegenstand von Leber ${ }^{1}$ ), Evetzky ${ }^{2}$ ), Reiss ${ }^{3}$ ) ausführlich diskutiert wurde.

In allen den früher angeführten Fällen - mein Fall II kommt hier natürlich, da Phthisis bulbi bestand, nur teilweise in Betracht - findet sich übereinstimmend ein unverhältnismässig frühzeitiges Auftreten der extraokularen Neubildung, d. h. nach der üblichen Anschauung ein ausserordentlich frühzeitiger Durchbruch der intraokularen Geschwulstbildung nach aussen, so frühzeitig, dass dieselbe eintreten konnte, noch bevor der Kranke von seinem Leiden überhaupt Kenntnis batte und bevor irgend eine der die Anwesenheit eines intraokularen Tumors schon nach kurzer Zeit begleitenden sekundären Veränderungen - Netzhautabhebung, Sekundärglaukom - aufgetreten war. Auf diesen Umstand muss viel mehr Gewicht gelegt werden, als auf das relative Grössenverhältnis der intraokularen und extraokularen Geschwulst. Das relativ langsame Wachstum der intraokularen Geschwulst ist ja meist darauf zurückzuführen, dass zufolge der Unterbindung der zuführenden Blutgefässe durch die Geschwulstbildung selbst, infolge der Durchwachsung und Umwachsung der Emissarien, immer beträchtliche Ernährungsstörungen in dem intraokularen Tumor auftreten, welche zu Nekrosen des Tumorgewebes, in jedem Falle aber

1) Leber und Krahnstöver, Über die bei Aderhautsarkomen vorkommende Phthisis des Augapfels. v. Graefe's Arch. f. Ophthalm. Bd. XLV.

2) Evetzky, Weitere Studien über Aderhantsarkome. v. Graefe's Arch. f. Ophthalm. Bd XLV. 3. S. 563.

s) W. Reis, Zur Differentialdiagnose der mit Phthisis bulbi kombinierten Sarkome. Arch. f. Angenheilk. Bd. L. S. 20. 
zu einem langsameren Tempo der Wucherung Anlass geben. Ganz anders verhält es sich bei den extraokularen Neubildungen, bei denen Zufuhr und Abfuhr der Ernährungsflüssigkeiten vollständig ungehindert vonstatten gehen kann.

So sehen wir auch in meinem zweiten Falle die intraokulare Neubildung fast vollständig nekrotisieren. Dieser Fall hat daher für die weitern Deduktionen nicht jene Bedeutung, wie neben den in der Literatur vorfindlichen Beobachtungen mein erster Fall. Trotz des Fehlens jeglicher Erscheinungen regressiver Metamorphose ist hier die intraokulare Neubildung, welche erst bemerkt wurde, als die extraokulare schon eine wesentliche Grösse erreicht hatte, bis zur Ausführung der Enucleation unverhältnismässig klein geblieben.

Dies muss uns dann die Vermutung nahe legen, dass in unsern Fällen schon in dem allerersten Anfang der Geschwulst bestimmte Momente gelegen sein müssen, welche den eigenartigen Verlauf vorausbedingen. Diese Momente suche ich in erster oder einziger Linie in der Lokalisation des ersten Geschwulstknöt. chens. Die Möglichkeit, dass der primäre Herd der Geschwulst episkleral sei und die Neubildung, während sie sich episkleral ausbreitet, längs eines Emissariums nach innen durch die Sklera durchwachse und $\mathrm{zu}$ intraokularer Neubildung führe, glaube ich ohne weiteres von der Hand weisen zu können. Es spricht dagegen in erster Linie die Erfabrung, dass bei allen Arten orbitaler Neubildungen, insbesondere Sarkomen und in geringerem Grade auch bei epibulbären Carcinomen, die Sklera, wie die Duralscheide des Sehnerven der Ausbreitung der Neubildung einen grossen Widerstand entgegensetzt. Dagegen erscheint es mir äusserst naheliegend anzunehmen, dass in diesen Fällen die Neubildung selbst intraskleral, d. h. in dem pigmentierten, die Emissarien begrenzenden Gewebe aufgetreten sei.

Unter dieser Annahme ist es verständlich, wieso entweder gleichzeitig extra- und intraokulare Neubildung in Erscheinung treten können, welche dann von vornherein mit Rücksicht auf die schon angeführten, jeder von beiden zukommenden besondern Ernährungsverhältnisse ab initio einen beträchtlichen Grössenunterschied zu Gunsten der extraokularen Neubildung aufweisen können, oder in anderen Fällen die intraokulare Neubildung erst deutlich 
sich manifestieren kann, wenn die extraokulare schon ganz beträchtliche Dimensionen erreicht hatte.

Kann man sich nicht entschliessen, diese Lokalisation des primären Geschwulstknotens anzunehmen, so müsste man jedenfalls den Ausgangspunkt der Geschwulst in den oberflächlichen Chorioidealschichten (Lamina fusca) suchen und könnte das Eigenartige des Verlaufes dadurch bedingt sehen, dass dieser Herd gerade dicht an einem Emissarium sich befand, wodurch dann das rasche Wachstum der Neubildung durch die Sklera, eben längs des Emissariums sich erklären könnte. Mir erscheint aber diese letztere Erklärungsweise immerhin gezwungener, als die vorher angeführte. Jedenfalls wäre, falls analoge Fälle zur Beobachtung kommen, ganz besonders künftig darauf zu achten, in welcher Art die Verbindung zwischen extraokularer und intraokularer Neubildung hergestellt ist, um dadurch vielleicht doch nähere Anhaltspunkte zu gewinnen für die von mir angenommene Lokalisation des ersten Geschwulstknötchens.

Der erst angeführte Fall erlaubt uns noch einer zweiten Frage, welche besonders in jüngster Zeit wieder grosses Interesse erweckt hat, etwas näher zu treten. Es ist vor nicht langer Zeit durch Elschnigl) darauf hingewiesen worden, dass bei intraokularen Geschwulstbildungen, wenn sie vor dem Eintreten sekundärer Netzhautablösung und Drucksteigerung zur anatomischen Untersuchung kommen, eine der beim Tumor cerebri vorkommenden Stauungspapille nahezu identische Neuritis am Sehnerveneintritte beobachtet wird. Auch in meinem Falle ergibt sich aus der Beschreibung des anatomischen Befundes ein gleiches Verhalten. Dieser bei Tumor vorkommenden intraokularen Neuritis kommt deswegen eine besondere Bedeutung zu, weil daraus Rückschlüsse auf die Entstehung der Stauungspapille bei Hirntumoren, die ja immer noch nicht vollständig überzeugend erwiesen ist, gezogen werden können. Hirschberg hat zuerst das Vorkommen einer der Stauungspapille gleichen intraokularen Neuritis bei intraokularen Erkrankungen, und zwar nach perförierender Fremdkörperverletzung beobachtet und als perforative Neuritis angesprochen. Elschniga hat dann in seiner letzten Mitteilung über die Pathogenese der Stauungspapille bei intrakraniellen Erkrankungen zuerst

1) Elschnig, Bemerkungen zu Kampherstein, Beitrag zur Pathologie und Pathogenese der Stauungspapille. Klin. Monatsbl. f. Augenheilk. 1904. Nov.

2). Elschnig, Die Pathogenese der Staungspapille bei Hirntumor. Wiener klin. Rundschau. 1902. Nr. 1, 2 u. 3.

v. Graefe's Archiv für Ophthalmologie. LXIII. 1. 
festgestellt, dass bei jeglicher Art von intraokularer Entzündung und intraokularer Neubildung Neuritis optici unter dem Bilde der Stauungspapille vorkomme. Auch in jenen Fällen, in welchen es bereits zur Aushöhlung durch Glaukom gekommen ist, lässt sich in dem peripheren Ende des Sehnerven eine mehr oder weniger intensive interstitielle Neuritis in jedem Falle intraokularer Neubildung konstatieren. Gerade das Auftreten von Neuritis optici bei intraokularen Geschwülsten, welche nicht direkt den Sehnerven in Mitleidenschaft ziehen, scheint den Beweis dafür zu liefern, dass doch, wie ja die moderne Entzuindungstheorie der Pathogenese der Stauungspapille bei intraokularen Erkrankungen es verlangt, der Tumor, bzw. seine Stoffwechselprodukte, phlogogene Wirkung besitzen.

Auf ein zweites Moment möchte ich auch noch an dieser Stelle hinweisen, nämlich auf das Auftreten oder Ausbleiben sekundärer Netzhautablösung bei intraokularem Sarkom. In meinem ersten Falle, wie ja auch in den aus der Literatur angeführten analogen Fällen ergibt sich, dass das Auftreten einer sekundären Netzhautablösung wenigstens durch lange Zeit hindurch verhindert wird, wenn die Netzhaut in grösserer Ausdehnung mit der Neubildung verwachsen ist. Das Warum ist hier leicht erklärlich. Eine seröse Netzhautablösung bei Tumor kann ja nur dann entstehen, wenn die seröse Absonderung, die aus dem Tumor oder der ihn deckenden Chorioidea stattfindet, sich zwischen letzterer und Netzhaut ansammeln kann. Es ist ungemein naheliegend, gerade für die weitere Zunahme der Netzhautablösung dann, im Sinne der Raehlmannschen Theorie der Entstehung der Netzhautablösung, osmotische Vorgänge zwischen Glaskörper und subretinaler Flüssigkeit verantwortlich zu machen. Dafür spricht auch ein ganz eigenartiger Befund, wie er in meinem ersten. Falle zu erheben war. Es war hier in einer grossen Ausdehnung in der den Tumor deckenden Netzhaut zu einer Spaltung der Netzhant in zwei Lamellen - also zu einer Art Cystenbildung - gekommen. Ich möchte hierauf noch mit wenigen Worten eingehen, da dieser eigenartige und soweit mir die Literatur bekannt ist, neue Befund, auf ein zuerst von Bruns ${ }^{1}$ ) beobachtetes Verhalten der Netzhaut zum Chorioidealtumor, ein aufklärendes Licht wirft. Bruns fand in zwei seiner Fälle die Netzhaut in zwei Lamellen - also analog wie bei mir — gespalten, wobei

1) O. Bruns, Beiträge zur Lehre von den Aderhautsarkomen. v. Graefe's Arch. f. Ophtbalm. Bd. LTV. 3. 
die beiden Lamellen den Tumor kapselartig einhüllen. Bruns erklärte dies so, dass die Netzhaut zuerst mit der Aderhaut verwachsen war, dann die Lamina vitrea chorioideae und die mit ibr verwachsene Netzhaut, nämlich Pigmentepithel und die äussere Körnerschicht derselben, soweit sie mit der Lamina vitrea chorioideae unlöslich verbunden war, platzte, worauf der Tumor zwischen die sich so bildenden zwei Blätter der Netzhaut, etwa in die Zwischenkörnerschicht, hineinwuchs.

Mein Befund lehrt, dass diesem Einwachsen unbedingt die Spaltung der Netzhaut in zwei Lamellen vorausgeht, worauf offenbar erst nachträglich, nach Berstung des äussern mit der Lamina vitrea chorioideae verwachsenen Blattes, die Tumormassen in den präformierten cystenähnlichen Hohlraum hineinwuchern. Die primäre Entstehung der Spaltung der Netzhaut in zwei Lamellen lässt sich aus meinem histologischen Befunde leicht ersehen. Sie beginnt, wie an den beiden Randteilen des cystenähnlichen Hohlraumes zu ersehen, als eine besondere, der cystoiden Entartung der senilen Netzhaut ähnliche Degeneration der mittlern Netzhautlagen; ist es einmal zur Bildung eines cystischen Hohlraumes gekommen, so lässt sich sein auffallendes Grössenwachstum wohl am leichtesten durch osmotische Vorgänge im Sinne Raehlmanns erklären.

\section{Resumé.}

Aus meinen vorstehenden Untersuchungen lassen sich folgende Sätze hervorheben.

I. Das Auftreten sekundärer Netzhautabhebung bei intraokularem Sarkom wird dadurch verhindert oder wenigstens verzögert, dass die Geschwulst in ausgedehnterem Bereiche mit der Netzhaut verwächst, welche Verwachsung zum Teil wenigstens durch Wucherung des Pigmentepithels zu stande gekommen scheint. (Ähnlich wie bei Heilung cler Netzhautablösung.)

II. Bei intraokularen Tumoren, die noch nicht zu sekundärem Glaukom geführt haben, findet sich eine der sog. Stauungspapille ähnliche intraokulare Neuritis.

III. Es gibt eine Anzahl seltener Fälle, bei welchen ausserordentlich frühzeitig, mit Überspringen wenigstens des zweiten Stadiums, also vor dem Auftreten der Netzhautablösung und vor dem sekundären Glaukom bereits extraokulare Neubildung auftritt.

Klinische und anatomische Tatsachen erfordern die Annahme, dass dieser eigenartige Verlauf des Wachstums 
durch eine eigenartige Lokalisation des primären Geschwulstherdes bedingt sei, und legen nahe, anzunehmen, dass der primäre Sitz der Neubildung in diesen Fällen intraskleral gelegen sein könne.

Letztere Annahme ist natürlich nur für Fälle zulässig, in denen der extraokulare Geschwulstknoten, seiner histologischen Beschaffenheit nach, ältern Datums zu sein scheint, und der intraokulare völlig frei von nekrotischen Veränderungen ist.

Erklärung der Abbildungen auf Taf. V, Fig. 1-5.

Fig. 1. Übersichtsbild vom Fall I. - Hämatoxylin-Eosinfärbung (halbschematisch). Fig. 2. Sehnervenpapille and hinterer Rand der Aderhantgeschwulst und Netzhautcyste in Fall I. Hämatoxylin-Eosinfärbung.

Fig. 3. Vorderer Rand der Aderhautgeschwulst (links im Bilde ist vorn, rechts ist hinten).

Fig. 4. Vorderes Ende der Cyste bzw. Netzhautspalte (links im Bilde ist hinten, rechts ist vorn).

Fig. 5. Übersichtsbild von Fall II. 
$\stackrel{-}{5}$

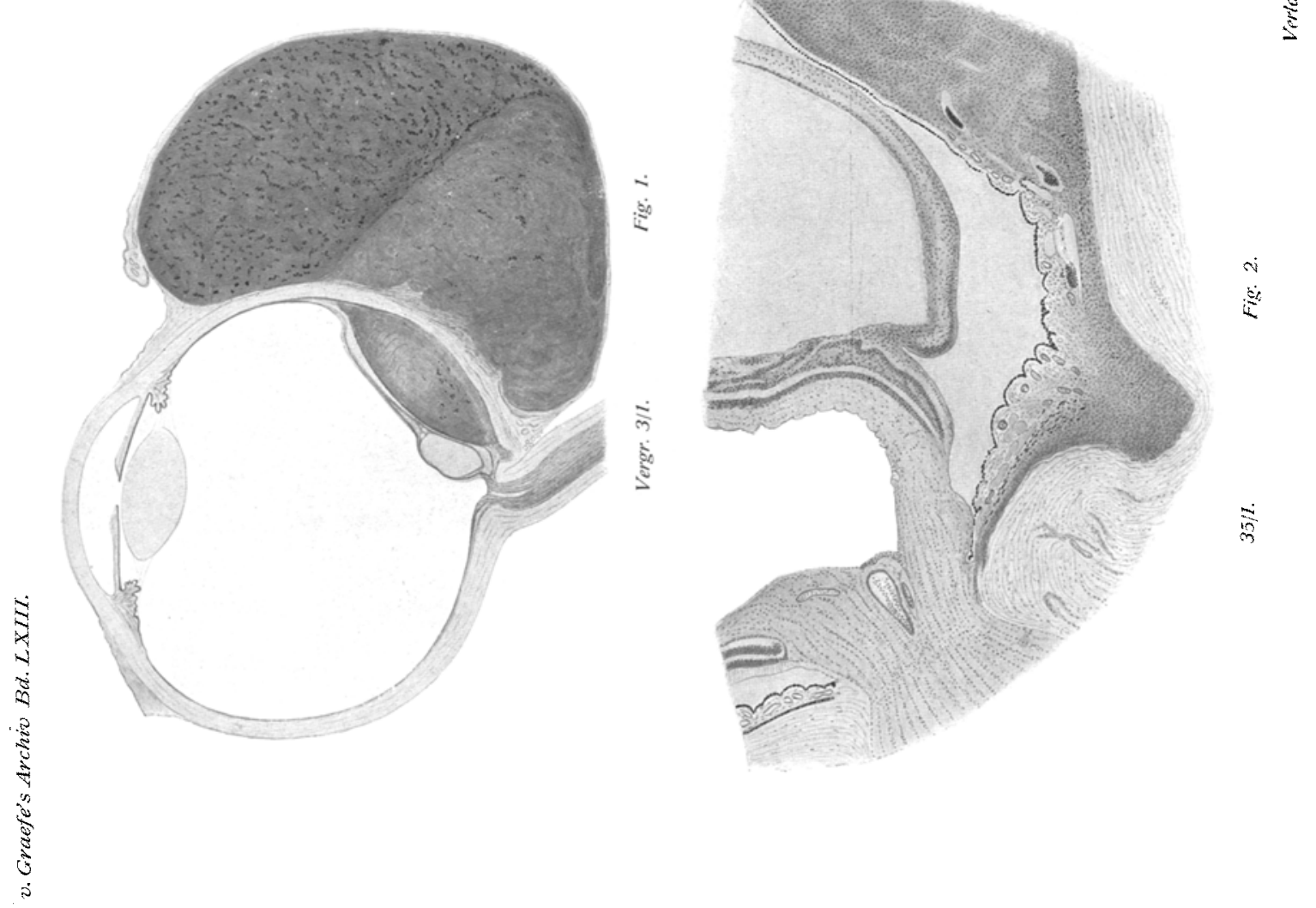

